\title{
Commas, Part 1
}

\author{
Mark B. Mycyk
}

Published online: 11 March 2010

(C) American College of Medical Toxicology 2010

\section{Commas, Part 1: Resolving Serial Poisoning Ambiguity with the Serial Comma}

The serial comma, also known as the Oxford comma or Harvard comma, has become an endangered species in writing. It was traditionally used before the final conjunction in a list of three or more items to provide clarity and visual symmetry on the printed page (e.g., "arsenic, cyanide, and strychnine"). To save space, the serial comma has virtually disappeared from most forms of writing. Although hardly missed, this endangered comma can still help writers avoid unintended ambiguity in some lists of items.

Example \#1 The fellowship candidate credited many people, particularly his parents, Paracelsus and Michael Swango, for inspiring his keen interest in poisons.

The list of three in example \#1 is correctly punctuated, but the meaning of the sentence remains ambiguous. The candidate intends to credit three separate influences: his parents and Paracelsus and Michael Swango. However, one can easily misread the sentence and think the candidate's parents are Paracelsus and Michael Swango. Adding a seemingly innocuous serial comma can eliminate all ambiguity.

Example \#2 The fellowship candidate credited many people, particularly his parents, Paracelsus, and Michael Swango, for inspiring his keen interest in poisons.

All writers hoping to resolve serial ambiguity should not forget about the endangered but powerful serial comma.

M. B. Mycyk $(\bowtie)$

Cook County Hospital, Toxikon Consortium,

Chicago, IL, USA

e-mail: mmycyk@ccbh.org 